\title{
NEAR OBSERVATIONAL EQUIVALENCE AND \\ FRACTIONALLY INTEGRATED PROCESSES
}

Marmol F. and Reboredo, J.C.

98-38

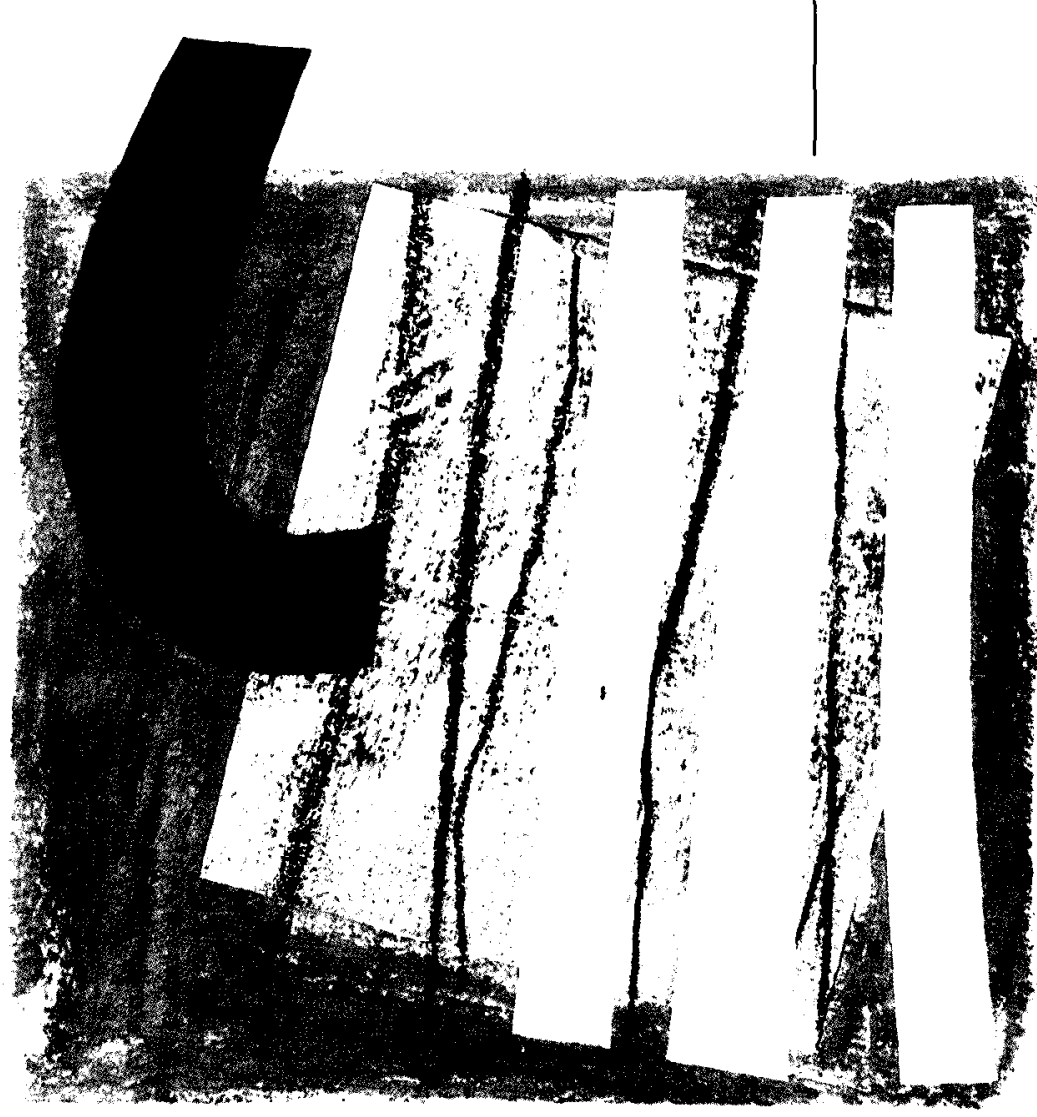

$\frac{0}{\frac{1}{2}}$

$\frac{\frac{1}{2}}{\frac{1}{2}}$ 
Working Paper 98-38

Statistics and Econometrics Series 18

June 1998
Departamento de Estadística y Econometría

Universidad Carlos III de Madrid

Calle Madrid, 126

28903 Getafe (Spain)

Fax (341) 624-9849

\title{
NEAR OBSERVATIONAL EQUIVALENCE AND \\ FRACTIONALLY INTEGRATED PROCESSES
}

Marmol F. and Reboredo, J.C. *

\begin{abstract}
The aim of this paper is to study the presence of nearly observationally equivalence problems in fractionally integrated processes. In order to illustrate our results, by means of a Monte Carlo study, we analyse the finite sample behaviour of the usual Durbin-Watson statistic in a regression between two independent nonstationary fractionally integrated processes wiht $M A(1)$ innovations.
\end{abstract}

Key Words

Near observational equivalence; fractional integration and cointegration; Durbin-Watson statistic.

*Department of Statistics y Econometrics, Universidad Carlos III de Madrid, e-mail: fmarmol@est-econ.uc3m.es; Department of Economics, Universitat Autònoma de Barcelona. We are grateful to Anindya Banerjee, Juan J. Dolado and Jesús Gonzalo for useful comments and suggestions. The first author acknowledges grant from the Training and Mobility of Researches Program. The second author tanks the DGICYT PB94-0709 for financial support. J.E.L. Classification: C12, C15, C22. 


\title{
NEAR OBSERVATIONAL EQUIVALENCE AND FRACTIONALLY INTEGRATED PROCESSES *
}

\author{
Francesc $\mathrm{Marmol}^{+}$ \\ Department of Statistics and Econometrics, Universidad Carlos III de Madrid \\ $\&$ \\ Juan C. Reboredo \\ Department of Economics, Universitat Autònoma de Barcelona
}

First Version, September 1997

Revised Version, May 1998

\begin{abstract}
SUMMARY
The aim of this paper is to study the presence of nearly observationally equivalence problems in fractionally integrated processes. In order to illustrate our results, by means of a Monte Carlo study, we analyse the finite sample behaviour of the usual Durbin-Watson statistic in a regression between two independent nonstationary fractionally integrated processes with $M A(1)$ innovations.
\end{abstract}

Keywords: Near observational equivalence; Fractional integration and cointegration; Durbin-Watson statistic.

J.E.L. Classification: C12, C15, C22.

\footnotetext{
- We are very grateful to Anindya Banerjee, Juan J. Dolado and Jesús Gonzalo for useful comments and suggestions. The first author acknowledges grant from the Training and Mobility of Researches Program. The second author thanks the DGICYT PB94-0709 for financial support.

Address for correspondence: Francesc Marmol, Department of Statistics and Econometrics, Universidad Carlos III de Madrid, 28903 Getafe, Madrid, Spain. E-mail: fmarmol@est-ccon.uc3m.es. Phone: + (34) 162498 63. Fax: + (34) 16249849.
} 


\section{INTRODUCTION}

It is a well known fact that the customary unit root and cointegration tests can be affected by size distortions, particularly when the $I(1)$ series have large $M A$ error terms (see, e.g., Molinas, 1986, Schwert, 1989). This issue has deserved much attention in the $I(1) / I(0)$ literature since such tests do not seem to work well in order to correct this model-misspecification problem. This is due to the fact that for any unit root process (respectively $I(0)$ ), it can be proved that there exists a stationary process (respectively $I(1)$ ) which will be impossible to distinguish from the unit root (respectively $I(0)$ ) representation for any given sample size. In other words, $I(1)$ and $I(0)$ are nearly observationally equivalent in Faust's (1993) sense

Likewise, there has been an increasing empirical evidence in recent years supporting the assumption that many macroeconomic and financial time series achieve stationarity after applying a fractional filter. Most important, there are many reasons to believe that the paths of some relevant economic series cannot only be well approximated by fractionally integrated processes, but also that they do contain moving average components. In this respect, several studies have obtained fractionally integrated processes with $M A$ components when modelling the behaviour of many important economic variables. A partial list of these variables includes the growth rate of US real GNP (Sowell, 1992), annual real per capita US GDP (Cheung, 1993), annual bond yields (Crato and Rothman, 1994), inflation series (Baillie et al., 1995), Standard \& Poor 500 index series (Hauser and Kunst, 1995) or traded good prices (Barkoulas et al., 1996).

How frequently do we observe fractionally integrated processes with large $M A$ innovations? For instance, as an empirical illustration, using exact maximum likelihood methods, Barkoulas et al. (1996) show that the inflation rates of traded good prices of the import series for US (covering the period 1969:1 to 1994:12) and Canada (covering 
the period 1957:1 to $1994: 12$ ) have memory parameters (in parenthesis the standard errors) equal to $0.862(0.046)$ and $0.645(0.09)$, with innovations driving by $M A(1)$ processes with parameters $-0.635(0.046)$ and $-0.79(0.066)$, respectively.

Consequently, it appears as a relevant issue to address the question of the existence and influence of near observational equivalence in the more general fractional set-up. For this, in Section II of the paper we make precise a sense in which data are uninformative about the memory parameter of the time series by extending the notion of near observational equivalence proposed by Faust (1993) to the fractional framework. In Section III we provide an illustration of the pitfalls that can arise in this framework by providing some experimental evidence on the behaviour of the Durbin-Watson statistic in a linear regression between the levels of two independent nonstationary fractionally integrated processes with $M A$ components. Concluding remarks are given in Section IV.

\section{NEAR OBSERVATIONAL EQUIVALENCE WITH}

\section{FRACTIONAL PROCESSES}

In this section, we shall give a precise meaning to the notion of near observational equivalence with fractionally integrated processes. As is well-known, a time series $y_{t}$ is said to be fractionally integrated of order $d$, denoted $y_{t} \sim F I(d)$ if it becomes weakly stationary after differentiating $d$ times, and the degree of differentiation or memory parameter, $d$, is a real number.

Throughout the paper we shall assume that the series of interest, $y_{t}$, follows a fractionally integrated process with Wold representation

$$
\Delta^{d} y_{t}=c(L) \varepsilon_{t}
$$


where $d>-1, c(L)=\sum_{j=0}^{\infty} c_{j} L^{j}$ is 1-summable so that $c(1)=\sum_{j=0}^{\infty} c_{j}$ is well-defined, $\varepsilon_{t}$ is white noise with zero mean and $E\left(\varepsilon_{1}^{2}\right)=\sigma_{\varepsilon}^{2}<\infty$ and where, without loss of generality, the initial conditions are set equal to zero.

Under these assumptions, to each $c(L)$ it can be associated the infinite sequence $\aleph=\left\{c_{0}, c_{1}, c_{2}, \ldots\right\} \in \ell^{1}$, where $\ell^{\prime}$ denotes the space of absolutely summable sequences. Following Faust (1993), we shall refer to $\aleph$ as the $M A$ representation of the process with lag polynomial $c(L)$. Thus, the parameters of our model are given by $\theta=\left(\aleph ゙, \sigma_{c}^{2}\right) \in \Theta, \Theta=\ell^{1} \times \mathfrak{R}_{+}$. Given this parameterization, for each sample size $T$ and each $\theta \in \Theta$ and with $\Im(\cdot \mid \theta)$ denoting the corresponding distribution function, following Faust (1993), we say that the sequence of structures parameterized by $\left\{\theta_{k}\right\}$ has nearly observationally equivalent members to a structure $\theta_{r}$ if for any fixed $T$, $\Im\left(\cdot \theta_{k}\right) \Rightarrow_{k} \Im\left(\mid \theta_{r}\right)$, where " $\Rightarrow$ " means convergence is distribution of the underlying random variables. Hence, with continuous distributions, near equivalence of members of the sequence $\left\{\theta_{k}\right\}$ to $\theta_{r}$ implies that for any $\varepsilon>0$ we can find a $k$ such that $\Im\left(\gamma \mid \theta_{k}\right)-\Im\left(\gamma \mid \theta_{r}\right)<\varepsilon$ for all $\gamma$. Thus, the corresponding underlying processes are said to be nearly observationally equivalents in the previous sense.

Proposition 1. For any sample size, if there exists values of the sequence of coefficients in $\aleph$ making $c(1)$ be arbitrarily close to zero, then the fractionally integrated process $y_{t}$ of Equation (1) with memory parameter $d$ is nearly observationally equivalent to a fractionally integrated process with memory parameter $d-1$ and lag polynomial $\widetilde{c}(L)=\sum_{j=0}^{\infty} \widetilde{c}_{j} L^{j}$, with $\widetilde{c}_{j}=-\sum_{k=j+1}^{\infty} c_{k}, \sum_{j=0}^{\infty}\left|\widetilde{c}_{j}\right|<\infty$. 
Proof: Under the assumption of 1-summability, we know that

$$
c(L)=c(1)+(1-L) \widetilde{c}(L)
$$

where $\widetilde{c}(L)=\sum_{j=0}^{\infty} \widetilde{c}_{j} L^{j}, \widetilde{c}_{j}=-\sum_{k=j+1}^{\infty} c_{k}, \sum_{j=0}^{\infty}\left|\widetilde{c}_{j}\right|<\infty$ and $|c(1)|<\infty$. Thus,

$$
\Delta^{d} y_{t}=c(1) \varepsilon_{t}+(1-L) \widetilde{c}(L) \varepsilon_{t}
$$

so that

$$
y_{t}=c(1) \Delta^{-d} \varepsilon_{t}+\widetilde{c}(L) \Delta^{1-d} \varepsilon_{t}=c(1) y_{1 t}+\widetilde{c}(L) y_{2 t}=y_{1 t}^{*}+y_{2 t}^{*}
$$

say, with $y_{1 t}^{*}, y_{2 t}^{*}$ denoting fractionally integrated processes of order $d$ and $d-1$, respectively. Notice that if $c(1)=0$ then $y_{t}$ becomes equal to $y_{2 t}^{*}$, a fractionally integrated process of order $d-1$ with $M A$ representation $\widetilde{\aleph}=\left\{\widetilde{c}_{0}, \widetilde{c}_{1}, \widetilde{c}_{2}, \ldots\right\} \in \ell^{\prime}$. Thus, assuming the existence of a sequence of coefficients in $\aleph$ such that $c(1)$ be arbitrarily close to zero (in the $\ell^{2}$ norm), then the proof of the proposition follows directly from the denseness of sequences with any sum in $\ell^{1}$ and Proposition 1 given in Faust (1993). Q.E.D.

Proposition 1 shows how the possible existence of near observational equivalence problems in the $I(1) / I(0)$ literature are not exclusive of this family of processes but also extends to the fractionally integrated case. Notice, however, that by imposing the closeness of $c(1)$ to zero, which is a convenient and logical assumption in order to study size problems, we are constraining the class of fractionally integrated models under consideration. Thus, for example, in the particular $\operatorname{FIMA}(d, 1)$ case,

$$
\Delta^{d} y_{t}=(1-\vartheta L) \varepsilon_{t}
$$


where $c(L)=1-. L$ and $\widetilde{c}(L)=\vartheta$, expression (3) becomes

$$
y_{t}=(1-\vartheta) y_{1 t}+\vartheta y_{2 t}
$$

and for large $T$, the dynamics of the $y_{t}$ series is dominated by that of the fractional component $(1-\vartheta) y_{1 t}$, a fractionally integrated noise with the same memory parameter $d$ times the constant $(1-\vartheta)$. However, if $\vartheta$ is close to 1 , then in any finite sample, $y_{t}$ behaves essentially like the $y_{2 t}$ series, a fractionally integrated noise process with memory parameter $d-1$. Hence, the $\operatorname{FIMA}(d, 1)$ process is nearly observationally equivalent to a fractionally integrated noise process with memory parameter $d-1$. Notice that in the particular case where $d=1$ we have the classical identification problems between an $\operatorname{IMA}(1,1)$ and a white noise process.

Consider now the $\operatorname{ARFIMA}(1, d, 0)$ process

$$
(1-\rho L) \Delta^{d} y_{t}=\varepsilon_{t}, \quad|\rho|<1
$$

In this case, the $M A$ representation is given by

$$
\Delta^{d} y_{t}=\frac{1}{1-\rho L} \varepsilon_{t},
$$

so that $c(1)=(1-\rho)^{-1}, \widetilde{c}(L)=-\rho(1-\rho)^{-1}(1-\rho L)^{-1}$ and expression (3) becomes equal to

$$
y_{t}=\left(\frac{1}{1-\rho}\right) y_{1 t}-\left(\frac{\rho}{1-\rho}\right)\left(\frac{1}{1-\rho L}\right) y_{2 t} .
$$

For large $T$, the dynamics of $y_{t}$ is now dominated by that of the fractional component $(1-\rho)^{-1} y_{1 t}$, a fractionally integrated noise with the same memory parameter $d$ times the constant $(1-\rho)^{-1}$. However, in this case, there is no value of $\rho$ for which $y_{t}$ behaves essentially like the $y_{2 t}$ series. Consequently, the $\operatorname{ARFIMA}(1, d, 0)$ process is not 
nearly observationally equivalent to a fractionally integrated process with memory parameter $d-1$ and lag polynomial given by $\widetilde{c}(L)=-\rho(1-\rho)^{-1}(1-\rho L)^{-1}$. This is because in the $\operatorname{ARFIMA}(1, d, 0)$ case, $c(1)$ is uniformly bounded below by $\frac{1}{2}$ so that Proposition 1 does not applies.

Finally, it is direct to show that the general $\operatorname{ARFIMA}(p, d, q)$ family of processes, with $q>0$, satisfies Proposition 1 , since there always exists values of the coefficients in the $M A(q)$ polynomial such that $c(1)$ can be made (in $\ell^{2}$ sense) arbitrarily close to zero.

\section{TESTING FOR FRACTIONAL COINTEGRATION BY MEANS OF THE CRDW TEST}

In the context of testing for cointegration within the $I(1) / I(0)$ framework, one of the most popular test in the literature has been the so-called Cointegrating Regression Durbin-Watson (CRDW) test, first suggested by Sargan and Bhargava (1983). Assuming that the cointegrating regression of interest is composed by $I(1)$ processes, this test has as null hypothesis no cointegration, that is, that the innovation process follows also an I(1) process against the alternative of cointegration with the innovations now evolving as weakly stationary or $I(0)$ processes.

Under the null of spurious regression, Phillips (1986) proved that the Durbin-Watson $(D W)$ statistic converges in probability to zero, whereas under the alternative of cointegration, it is well-known that such statistic weakly converges to $2\left(1-\rho_{1}\right)$, with $\rho_{1}$ denoting the first-order autocorrelation of the innovations.

This dissimilar behaviour of the Durbin-Watson statistic, both under the null and under the alternative, forms the basis of the CRDW test. See, for instance, Engle and Granger 
(1987). At an experimental level, however, Granger and Newbold (1986, Table 6.4) and Molinas (1986) showed, by means of a small sample regression between two independent $\operatorname{IMA}(1,1)$ series, that for large values of the $M A$ parameters of the dependent variable the number of rejections approaches 100 .

In a similar manner, one could propose the use of the $C R D W$ test for testing for cointegration among nonstationary $\left(d>\frac{1}{2}\right)$ fractionally integrated processes. The idea of the test would be as follows. Consider, without loss of generality, the simplest case where we want to test for cointegration between two nonstationary fractionally integrated processes of order $d$. The null hypothesis, as in the standard $C R D W$ test, is no cointegration. Under this null, Marmol (1998a) proves that the $D W$ converges in probability to zero for all $d>\frac{1}{2}$. On the other hand, consider the alternative hypothesis of fractional cointegration with the innovation series following a stationary fractionally integrated process of order $\delta,|\delta|<\frac{1}{2}$. Under this alternative, Marmol (1998b) proves that the $D W$ statistic weakly converges to $2\left(1-\rho_{l}(\delta)\right)$, where $\rho_{1}(\delta)$ stands for the firstorder autocorrelation coefficient of the stationary fractionally integrated of order $\delta$ innovations. Notice that in the particular case where $d=1, \delta=0$ we recover the standard $C R D W$ cointegrating test.

Indeed, in the light of the results obtained in the preceding section, it appears of interest to perform a Monte Carlo study about the finite sample properties of such fractional $C R D W$ test when the data generating process is assumed to be composed by a bivariate system of independent nonstationary $\operatorname{FIMA}(d, 1)$ processes. For this, consider the linear regression model $y_{t}=\hat{\beta} x_{t}+\hat{u}_{t}$, where $\left\{y_{t}\right\}_{t=1}^{T}$ and $\left\{x_{t}\right\}_{t=1}^{T}$ are two independent nonstationary $\operatorname{FIMA}(d, 1)$ processes, where $\hat{\beta}$ and $\hat{u}_{t}$ denote the $O L S$ 
estimator of the slope coefficient and the corresponding OLS residuals, respectively. The sample size considered was set equal to 100 and the number of replications was 10,000 . The main results obtained for the empirically relevant case where $\frac{1}{2}<d<\frac{3}{2}$ are summarized in Tables 1 and 2. A further more complete set of experiments is available upon request. Table 1 shows the mean, range and standard deviation of the $D W$ statistic in the Monte Carlo simulations. Table 2 shows the percent of times the proposed fractional $C R D W$ test rejects the null hypothesis of no cointegration.

\section{TABLE 1}

Range, mean and standard deviation of the empirical DW statistic

\begin{tabular}{|c|c|c|c|c|c|}
\hline Value of $\vartheta$ & $d=0.51$ & $d=0.6$ & $d=0.8$ & $d=1$ & $d=1.2$ \\
\hline & \multicolumn{5}{|c|}{ range } \\
\hline 0.4 & $0.91-2.54$ & $0.50-2.41$ & $0.18-1.74$ & $0.05-1.47$ & $0.03-1.52$ \\
\hline 0.6 & $1.32-2.83$ & $0.99-2.58$ & $0.33-2.25$ & $0.09-2.01$ & $0.06-1.87$ \\
\hline 0.8 & $1.85-3.03$ & $1.70-2.95$ & $0.75-2.59$ & $0.32-2.23$ & $0.21-2.24$ \\
\hline 0.9 & $2.08-3.11$ & $2.00-3.02$ & $1.42-2.73$ & $0.68-2.59$ & $0.72-2.42$ \\
\hline \multicolumn{6}{|c|}{ mean } \\
\hline 0.4 & 1.6809 & 1.4057 & 0.7952 & 0.4526 & 0.4136 \\
\hline 0.6 & 2.1356 & 1.9158 & 1.2810 & 0.8072 & 0.7397 \\
\hline 0.8 & 2.4914 & 2.3630 & 1.8976 & 1.3404 & 1.3135 \\
\hline 0.9 & 2.5969 & 2.4990 & 2.1785 & 1.7557 & 1.7075 \\
\hline \multicolumn{6}{|c|}{ std. error } \\
\hline 0.4 & 0.2850 & 0.3112 & 0.3062 & 0.2404 & 0.2309 \\
\hline 0.6 & 0.2372 & 0.2657 & 0.3523 & 0.3191 & 0.3327 \\
\hline 0.8 & 0.1838 & 0.1950 & 0.2746 & 0.3596 & 0.3657 \\
\hline 0.9 & 0.1656 & 0.1731 & 0.2033 & 0.2721 & 0.2834 \\
\hline
\end{tabular}




\section{TABLE 2}

Percentage of CRDW rejections of the null hypothesis of no cointegration

\begin{tabular}{|cccccc|}
\hline Value of $\vartheta$ & $d=0.51$ & $d=0.6$ & $d=0.8$ & $d=1$ & $d=1.2$ \\
\hline \hline 0.4 & 93.9 & 87.2 & 63.3 & 56.5 & 54.9 \\
0.6 & 100 & 99.8 & 96.5 & 91 & 87.9 \\
0.8 & 100 & 100 & 100 & 99.9 & 99.9 \\
0.9 & 100 & 100 & 100 & 100 & 100 \\
& & & & & \\
$5 \%$ critical values & 1.2407 & 1.0519 & 0.6554 & 0.3763 & 0.3420 \\
\hline
\end{tabular}

$D G P: \Delta^{d} y_{t}=(1-\vartheta L) \varepsilon_{y t}, \Delta^{d} x_{t}=(1-\vartheta L) \varepsilon_{x t}, \varepsilon_{i t} \sim N I D(0,1), i=\{y, x\}, \vartheta=\{0.4,0.6,0.8,0.9\}$.

Estimated model: $y_{t}=\hat{\beta} x_{t}+$ res. $._{t} . T=100.5 \%$ critical values for each $d$ obtained from Monte Carlo simulation.

Looking at these tables and having in mind the theoretical results developed in Section II, the following comment clearly shows up. When $\frac{1}{2}<d<\frac{3}{2}$ it follows from Proposition 1 that the $y_{t}$ series (and hence, under the null of independence, the $\hat{u}_{t}$ series) will be nearly observationally equivalent to a stationary fractionally integrated process of order $-\frac{1}{2}<d^{*}<\frac{1}{2}$. In this case, and following Marmol (1998b), we know that the $D W$ statistic weakly converges to $2\left(1-\rho_{1}\left(d^{*}\right)\right)$. Thus, when $\frac{1}{2}<d<\frac{3}{2}$ the near observational equivalence leads the $D W$ statistic to belong to the rejection region of the test implying the rejection of the null hypothesis in virtually all cases. This conclusion is clear when comparing the $5 \%$ critical values in the last row of Table 2 with the range and mean values of the empirical $D W$ statistic in Table 1.

\section{CONCLUSIONS}

It is a well established result in the unit root literature that the so-called near observational equivalence causes very important size distortions in the unit root tests. In this paper we have proved, both at a theoretical level and through Monte Carlo 
simulations, how these identification problems also extend to the class of the fractionally integrated processes.

Theoretically, we prove the existence of near observational equivalence in fractionally integrated processes for which the long-run impact multiplier $c(1)$ in the corresponding $M A$ representation can be made arbitrarily close to zero. At the simulation level, we have illustrated this claim by studying the finite samples properties of the $C R D W$ test in a simple regression model between two independent nonstationary $\operatorname{FIMA}(d, 1)$ processes with large $M A$ parameters. The experiments fully agree with the theoretical findings and illustrate the importance of carefully considering the specific nature of the short-run components of the processes of interest.

\section{REFERENCES}

BAILLIE, R.T., CHUNG, C.F. and TIESLAU, M.A. (1995). "Analyzing Inflation by the Fractionally Integrated ARFIMA-GARCH Model", Journal of Applied Econometrics 11 , $23-40$

BARKOULAS, J.T., BAUM, C.F. and OGUZ, G.S. (1996). "Stochastic Long Memory in Traded Goods Prices", mimeo, Boston College.

CHEUNG, Y.W. (1993). "Test for Fractional Integration: A Monte Carlo Investigation", Journal of Time Series Analysis 14, 331-345.

CRATO, N. and ROTHMAN, P. (1994). "Fractional Integration Analysis of Long-Run Behavior for US Macroeconomic Time Series", Economics Letters 45, 287-291.

ENGLE, R.F. and C.W.J. GRANGER (1987). "Co-integration and Error Correction: Representation, Estimation, and Testing", Econometrica 55, 251-276. 
FAUST, J. (1993). "Near Observational Equivalence and Unit Root Processes: Formal Concepts and Implications", Discussion Paper 447, International Finance Discussion Papers, Board of Governors of the Federal Reserve System.

GRANGER, C.W.J. and NEWBOLD, P. (1986). Forecasting Economic Time Series, $2^{\text {nd }}$ ed., Academic Press, New York.

HAUSSER, M.A. and KUNST, R.M. (1995). "Fractionally Integrated Models with $A R C H$ Errors", mimeo, University of Economics and Business Administration, Vienna

HOSKING, J.R.M. (1981). “Fractional Differencing", Biometrika 68, 165-176

MARMOL, F. (1998a). "Spurious Regression Theory with Nonstationary Fractionally Integrated Processes", Journal of Econometrics 84, 232-250.

MARMOL, F. (1998b). "Can We Distinguish Spurious from Cointegrating Regressions on the Basis of Standard OLS Statistics?", mimeo, Department of Statistics and Econometrics, Universidad Carlos III de Madrid

MOLINAS, C. (1986). "A Note on Spurious Regressions with Integrated Moving Average Errors", Oxford Bulletin of Economics and Statistics 48, 279-282.

PHILLIPS, P.C.B. (1986). "Understanding Spurious Regressions in Econometrics", Journal of Econometrics 33, 311-340.

SARGAN, D. and A. BHARGAVA (1983). "Testing Residuals from Least Squares Regression for Being Generated by the Gaussian Random Walk", Econometrica 51, 153 174

SCHWERT, G.W. (1989). "Test for Unit Roots: A Monte Carlo Investigation", Journal of Business and Economic Statistics 7, 147-159.

SOWELL, F.B. (1992). "Modeling Long Run Behavior with the Fractional ARIMA Model", Journal of Monetary Economics 29, 277-302. 